\title{
Adaptation and Validation of the Bangla Version of the Depression Literacy Questionnaire
}

SM Yasir Arafat ${ }^{1,3^{*}}$, Syed Faheem Shams ${ }^{2}$, Md. Hafizur Rahman Chowdhury ${ }^{3}$, Esmot Zarin Chowdhury ${ }^{1}$, Mohima Benojir Hoque ${ }^{1}$ and Mohammad Abdul Bari ${ }^{1}$

${ }^{1}$ Department of Public Health, ASA University Bangladesh, Dhaka, Bangladesh

${ }^{2}$ Department of Psychiatry, Uttara Adhunik Medical College, Dhaka, Bangladesh

${ }^{3}$ Department of Psychiatry, Bangabandhu Sheikh Mujib Medical University, Bangladesh

\begin{abstract}
Background: Depression is a common recurrent disorder linked to reduced quality of life, morbidity, and mortality. Bangladesh is a densely populated developing country and literacy on depression of this huge population is a must need to cope with the depressive disorder burden.
\end{abstract}

Objective: It was aimed to adapt and validate Bangla version of Depression Literacy Questionnaire (D - Lit Bangla) for the assessment of the status of knowledge regarding depression.

Method: The validation study was conducted at the department of public health, ASA University Bangladesh. Data were collected from May 2016 to July 2016 from 194 students by self-reporting final Bangla version of D-Lit questionnaire obtained by standard adaptation procedure. Samples were selected by convenient sampling and data were analyzed by Statistical Package of Social Science (SPSS) 16.0 and Microsoft Excel 2010 version software.

Result: The Cronbach's $\alpha$ was 0.77 which reflects the internal consistency of the reliability. After varimax rotation form of factor analysis- only one factor was extracted and factor analysis revealed significant commonalities between the items.

Conclusion: 20 items D-Lit Bangla is psychometrically valid and can be used for further researches as well as clinical settings.

Keywords: D-Lit Bangla; Depression literacy; Depression measurement; Validation study

Abbreviations: D-Lit: Depression Literacy; WHO: World Health Organization; CVI: Content Validity Index; KMO: Kaiser-MeyerOlkin; SPSS: Statistical Package of Social Science; MDG: Millennium Development Goal; GDP: Gross Domestic Product

\section{Introduction}

Depression is a common, chronic, recurrent, treatable disorder linked to diminished role functioning and quality of life, medical morbidity, and mortality [1-6]. It been ranked as the 4th leading cause of disability worldwide by World Health Organization (WHO) and again it is forecasted that by 2020 , it will be the second leading cause $[1,2,7]$. Symptoms comprised of low mood, lack of interest or pleasure, reduced energy, guilty feelings, disturbed sleep or appetite, and difficulties in attention and concentration [2-8]. Depression presents typically in the early to mid-20s, becomes chronic and recurrent and causes substantial impairments and at its worst, depression can lead to suicide as almost 1 million lives are lost yearly due to suicide $[1,2]$. The information on the prevalence of major depression does not exist for most countries; the available data indicate that there is wide variability in prevalence [1]. Depressive disorders are considered as major source of nonfatal disease and in developing countries it represents a key determinant of health related disability [7]. Bangladesh is a densely populous developing country having total population is about 160 million; achieved health related Millennium Development Goals (MDGs) significantly with a noticeable Gross Domestic Product (GDP) growth [9]. At present the number of total registered physician is about 82,500 for this huge population and most of the physicians are related with the primary health care in different ways [9]. The life time prevalence of Major Depressive Disorder is $4.6 \%$ in Bangladesh and a good level of depression literacy is needed to cope the burden of depression in Bangladesh [7,8]. Bangla is one of the seventh most widely spoken languages in the world and literacy on depression of this huge population is a must need to cope with the depressive disorder burden as well as to reduce the stigma on mental health [10-12]. So, it was aimed to adapt and validate Bangla version of Depression Literacy Questionnaire (D-Lit Bangla) for the assessment of the status of knowledge regarding depression in different aspects.

\section{Methods}

\section{Ethical aspects}

The researchers were duly concern about the ethical issues related to the validation. Before conducting the study ethical clearance was taken from the ethical review committee (ERC) of the ASA University Bangladesh. Permission was taken from the questionnaire developing author, Professor Kathleen M Griffiths as well informed written consent was taken from the subjects and standard approaches were followed to ensure the confidentiality of the information of the respondents.

\section{Instrument adaptation}

The adaptation to Bangla was performed according to the state-

*Corresponding author: SM Yasir Arafat, Department of Psychiatry, Bangabandhu Sheikh Mujib Medical University, Dhaka, Bangladesh, Tel: +88017132729; E-mail: arafatdmc62@gmail.com

Received: May 09, 2017; Accepted: June 02, 2017; Published: June 09, 2017

Citation: Arafat SMY, Shams SF, Chowdhury MHR, Chowdhury EZ, Hoque MB et al. (2017) Adaptation and Validation of the Bangla Version of the Depression Literacy Questionnaire. J Psychiatry 20: 412. doi:10.4172/2378-5756.1000412

Copyright: () 2017 Arafat SMY, et al. This is an open-access article distributed under the terms of the Creative Commons Attribution License, which permits unrestricted use, distribution, and reproduction in any medium, provided the original author and source are credited 
of-the-art procedure of forward- backward translation. 1 psychologist and 1 general person, both of them were native speakers of the Bangla and were fluent in English translated into Bangla first. The translated Bangla versions were compiled, and single Bangla forward version was created. Then this forward version was translated back into English by a professional translator with experience in medical translation and by 1 medical doctor who had not been involved in previous steps. The back-translated versions were then compiled and compared by the researcher and all the four versions were submitted to the expert committee formulated for the validation study. The expert committee formulated the questionnaire and then pretesting was conducted at a tertiary care hospital among 40 patients without any basis of diagnosis. The suggested changes were made accordingly based on the pretesting responses. Finally, the Bangla version of D-Lit was formulated [11,13$15]$.

\section{Design and subjects}

Validation study was conducted at Public Health department, ASA University Bangladesh [11,13-15]. Data were collected from May 2016 to July 2016 from 194 undergraduate and graduate students with the final Bangla version of D-Lit with convenient sampling. Self-reporting questionnaire was distributed among the respondents and responses were collected instantly. The respondents were the undergraduate students of the department of Pharmacy, English, Business Administration and graduate students of the department of Public Health; willing to participate in the study. Sample size was estimated on basis of 10:1 ratio of participants to item as different recommendations are available regarding the ratio with consideration of rules of thumb as participants to variables $2: 1$ to $20: 1[9,11,13-15]$. The questionnaire pertain twenty statements and the respondents were requested to report the appropriateness of each statements regarding depression in different aspects. Data were collected, checked, edited and managed properly and were analyzed in SPSS 16 version and Microsoft Excel Software 2010 version.

\section{Results}

\section{Socio-demographic characteristics of respondents}

In this D-Lit Bangla validation study, age of the respondents', gender, educational qualification, occupation, marital status, net family income, habitat, type of family, family members, history of treatment of depression of the respondents or family members, and any attachment with mental health services were considered as socio-demographic variables (Table 1). Age of the respondents was found as: Mean \pm SD (Range): $25.6 \pm 7.71$ (19-56) years.

Table 2 revealed the item characteristics of D-Lit Bangla before and after varimax rotation. Item- Total Correlation matrix showed the value with factor loading and communalities (Table 2).

\section{Reliability of D-Lit Bangla}

The internal consistency was measured by Cronbach's Alpha ( $\alpha)$ and the value was 0.77 .

(Cronbach's Alpha ( $\alpha$ ) ranges from 0 to 1 and a value of $\geq 0.70$ is considered as good reliability).

\section{Validity of D-Lit Bangla}

Face validity was systematically assured and assessed during the adaptation of the D-Lit Bangla questionnaire and at the time of data collection by the responses as well as review of the items with the depressed patients and Psychiatrists.
Content validity was systematically assured and assessed during the adaptation of the D-Lit Bangla questionnaire and with the Content Validity Index (CVI) after consultation with the Psychiatrists. Items having CVI $<0.8$ were dropped as well as further item addition was requested to the expert panel. Based on the expert committee review the items having numbers of 5, 10 and 18 were dropped on grounds of consideration of the cultural equivalence. Moreover, at item 13 the diseases Multiple Sclerosis was replaced by Asthma on the same ground. Lastly, based on the recommendation of the expert committee a new item was added in regards to the side effect profile of the antidepressants. So, from the original 22 item, 3 items were dropped and 1 new item was added after following the extensive procedures and total 20 item D-Lit Bangla scale was constructed.

\section{Construct validity}

Exploratory Factor Analysis the principal component with varimax rotation was performed to assess the construct validity. Kaiser- MeyerOlkin (K M O) and Barlett's test of Sphericity was applied to the fitness of data for factor analysis. The KMO and Barlett's Test of sampling adequacy was found as 0.75 where a value of $\geq 0.5$ is considered as a good sampling adequacy. Factor analysis and extraction revealed high commonalities between the items ranging from 0.41-0.74 (Table 2) and only one component was extracted after the varimax rotation.

\section{Discussion}

Psychometrics bears a very important role in Psychiatry, Public Health, Primary Health Care and other fields as well for measuring the behavioral aspects [9]. Globally depression is a hugely burdensome problem that needs adequate knowledge, care and supports from others to ensure the better quality of life. D-Lit was developed by K. M. Griffiths et al. [12], Italian version was validated by Kiropoulos et al. [16]; Greek version was validated by Gulliver et al. [17], validation of Arabic version was done [7]. It was aimed to validate the Depression Literacy (D -Lit) questionnaire in Bangla to assess and to improve the understanding of depression of the community people. Internal consistency of D-Lit Bangla was found 0.77 (Cronbach's alpha) which suggest good reliability and aligns with D-Lit original (0.70), Greek version (0.88), Italian (0.92) and Arabic (0.78) validation study [7,12,16,17]. The face validity was assessed during the back translation and others steps during preparation of the final D-Lit Bangla questionnaire before the data collection starts $[11,13,14]$. The content validity was assessed in the steps of back translation, expert review, expert committee opinion and dropping of 3 item as well as addition of 1 item was done after following the standard guidelines and finally D-Lit Bangla comprised of 20 items was finalized $[9,10]$. The corrected item-total correlations were (Table 2) also aligns with other especially Arabic [7]. Factor analysis of D-Lit Bangla revealed single factor instrument as the solution could not be rotated during the factor analysis and other validations studies were also found it as a unidimentional scale $[7,12,16,17]$.

\section{Conclusion}

The results from this validation study indicate good psychometric properties of the D-Lit Bangla. This D Lit Bangla instrument will be helpful to assess the level of knowledge regarding depression in multiple aspects and ultimately will help the physicians as well as the patients to cope with the burden of depression in Bangladesh. Data were collected from single university students in a city of the country; institutions of other regions and heterogeneous groups were not included and that might strengthen the findings. Larger multicentred studies involving heterogeneous respondents may help to discover the situation 
Citation: Arafat SMY, Shams SF, Chowdhury MHR, Chowdhury EZ, Hoque MB, et al. (2017) Adaptation and Validation of the Bangla Version of the Depression Literacy Questionnaire. J Psychiatry 20: 412. doi:10.4172/2378-5756.1000412

Page 3 of 4

\begin{tabular}{|c|c|c|c|c|c|}
\hline Gender & Frequency & Percentage & Residence & Frequency & Percentage \\
\hline Male & 103 & 53 & Town & 163 & 84 \\
\hline \multirow[t]{2}{*}{ Female } & 91 & 47 & Village & 31 & 16 \\
\hline & Marital Status & & & Department & \\
\hline Married & 81 & 42 & Public Health & 45 & 23 \\
\hline Unmarried & 103 & 53 & English & 54 & 28 \\
\hline \multirow[t]{2}{*}{ Not Mentioned } & 10 & 5 & Pharmacy & 38 & 20 \\
\hline & Family type & & Business & 57 & 29 \\
\hline Nuclear & 104 & 52 & & Educational Status & \\
\hline Joint & 90 & 48 & Undergraduate & 144 & 74 \\
\hline Total & 194 & 100 & Graduate & 30 & 26 \\
\hline
\end{tabular}

Table 1: Distribution of item characteristics of $\mathrm{D}$-Lit Bangla $(\mathrm{N}=194)$.

\begin{tabular}{|c|c|c|c|}
\hline Item & Item-Total Correlation & Factor loading & Communalities \\
\hline D-lit Q1 & 0.35 & 0.44 & 0.66 \\
\hline D-lit Q2 & 0.26 & 0.31 & 0.62 \\
\hline D-lit Q3 & 0.26 & 0.32 & 0.67 \\
\hline D-lit Q4 & 0.21 & 0.28 & 0.55 \\
\hline D-lit Q5 & 0.42 & 0.54 & 0.41 \\
\hline D-lit Q6 & 0.35 & 0.42 & 0.65 \\
\hline D-lit Q7 & 0.27 & 0.34 & 0.7 \\
\hline D-lit Q8 & 0.15 & 0.21 & 0.63 \\
\hline D-lit Q9 & 0.33 & 0.39 & 0.63 \\
\hline D-lit Q10 & 0.36 & 0.46 & \\
\hline D-lit Q11 & 0.46 & 0.57 & 0.35 \\
\hline D-lit Q12 & 0.39 & 0.49 & 0.65 \\
\hline D-lit Q13 & 0.27 & 0.35 & 0.73 \\
\hline D-lit Q14 & 0.3 & 0.39 & 0.57 \\
\hline D-lit Q15 & 0.24 & 0.33 & 0.7 \\
\hline D-lit Q16 & 0.41 & 0.52 & 0.47 \\
\hline D-lit Q17 & 0.41 & 0.55 & 0.5 \\
\hline D-lit Q18 & 0.38 & 0.52 & 0.74 \\
\hline D-lit Q19 & 0.45 & 0.57 & 0.56 \\
\hline D-lit Q20 & 0.49 & 0.61 & 0.52 \\
\hline
\end{tabular}

Table 2: Distribution of item characteristics of D-Lit Bangla ( $N=194)$.

appropriately and using the scale in daily practice will be benefited for both the caregiver and service provider of the depressive patients.

\section{Competing Interest}

Authors declare of having no conflict of interest.

\section{Acknowledgements}

Authors acknowledge Srijony Ahmed, Amin Andalib and Syed Reazur Rahman for their kind support. Authors thank Professor Kathleen Griffiths for permitting to validate the scale in Bangla and Monon Psychiatric Hospital for logistic support.

\section{Funding}

It was a self-funded study.

\section{References}

1. Kessler RC, Bromet EJ (2013) The epidemiology of depression across cultures. Am Annu Rev Public Heal 34: 119-38.

2. World Health Organization (2013) Depression, a global public health concern. World Health Organization Department of Mental Health and Substance Abuse.

3. American Psychiatric Association (2013) Diagnostic and statistical manual of mental disorders (5th edn). American Psychiatric Association, Washington, DC. pp: 155-188.

4. Cowen P, Harrison P, Burns T (2012) Shorter oxford textbook of psychiatry (6th edn). Oxford University Press, Oxford. pp: 205-250.
5. Sadock BJ, Sadock VA, Ruiz P (2015) Synopsis of psychiatry (11th edn). Wolter Kluwer, India. pp: 347-380.

6. Semple D, Smyth R (2013) Oxford handbook of psychiatry (3rd edn). Oxford University Press, Oxford. pp: 231-299.

7. Darraj HA, Mahfouz MS, Sanosi MR Al, Badedi M, Sabai A, et al. (2016) Arabic translation and psychometric evaluation of the depression literacy questionnaire among adolescents. Psychiatry J 2016: 8045262.

8. Mohit MA, Maruf MM, Ahmed H, Alam MT (2012) Depression and physical illnesses: An update. Bangladesh Med J 40: 53-58.

9. Arafat SMY (2016) Doctor patient relationship: An untouched issue in Bangladesh. Int J Psychiatry 1: 2.

10. https://www.ethnologue.com/statistics/

11. Arafat SMY (2016) Psychometric validation of the Bangla version of the patient-doctor relationship questionnaire. Psychiatry J 2016: 4.

12. Griffiths KM, Christensen H, Jorm AF, Evans K, Groves C (2004) Effect of webbased depression literacy and cognitive-behavioural therapy interventions on stigmatising attitudes to depression: A randomised controlled trial. $\mathrm{Br} \mathrm{J}$ Psychiatry 185: 342-349.

13. Arafat SMY, Chowdhury HR, Qusar MS, Hafez M (2016) Cross cultural adaptation \& psychometric validation of research instruments: A methodological review. J Behav Health 5: 129-136.

14. Arafat SMY (2016) Cross cultural adaptation \& psychometric validation of instruments: Step-wise description. Int J Psychiatry 1: 4. 
Citation: Arafat SMY, Shams SF, Chowdhury MHR, Chowdhury EZ, Hoque MB, et al. (2017) Adaptation and Validation of the Bangla Version of the Depression Literacy Questionnaire. J Psychiatry 20: 412. doi:10.4172/2378-5756.1000412

Page 4 of 4

15. Arafat SMY (2016) Validation study can be a separate study design. Int J Med Sci Public Heal 5: 2421-2422.

16. Kiropoulos LA, Griffiths KM, Blashki G (2011) Effects of a multilingual information website intervention on the levels of depression literacy and depression-related stigma in Greek born and Italian-born immigrants living in
Australia: A randomized controlled trial. J Med Internet Res 13: e34.

17. Gulliver A, Griffiths KM, Christensen H, Mackinnon A, Calear AL, et al. (2012) Internet based interventions to promote mental health help-seeking in elite athletes: An exploratory randomized controlled trial. J Med Internet Res 14 e69. 